\title{
sugerencias al pliego de condiciones
}

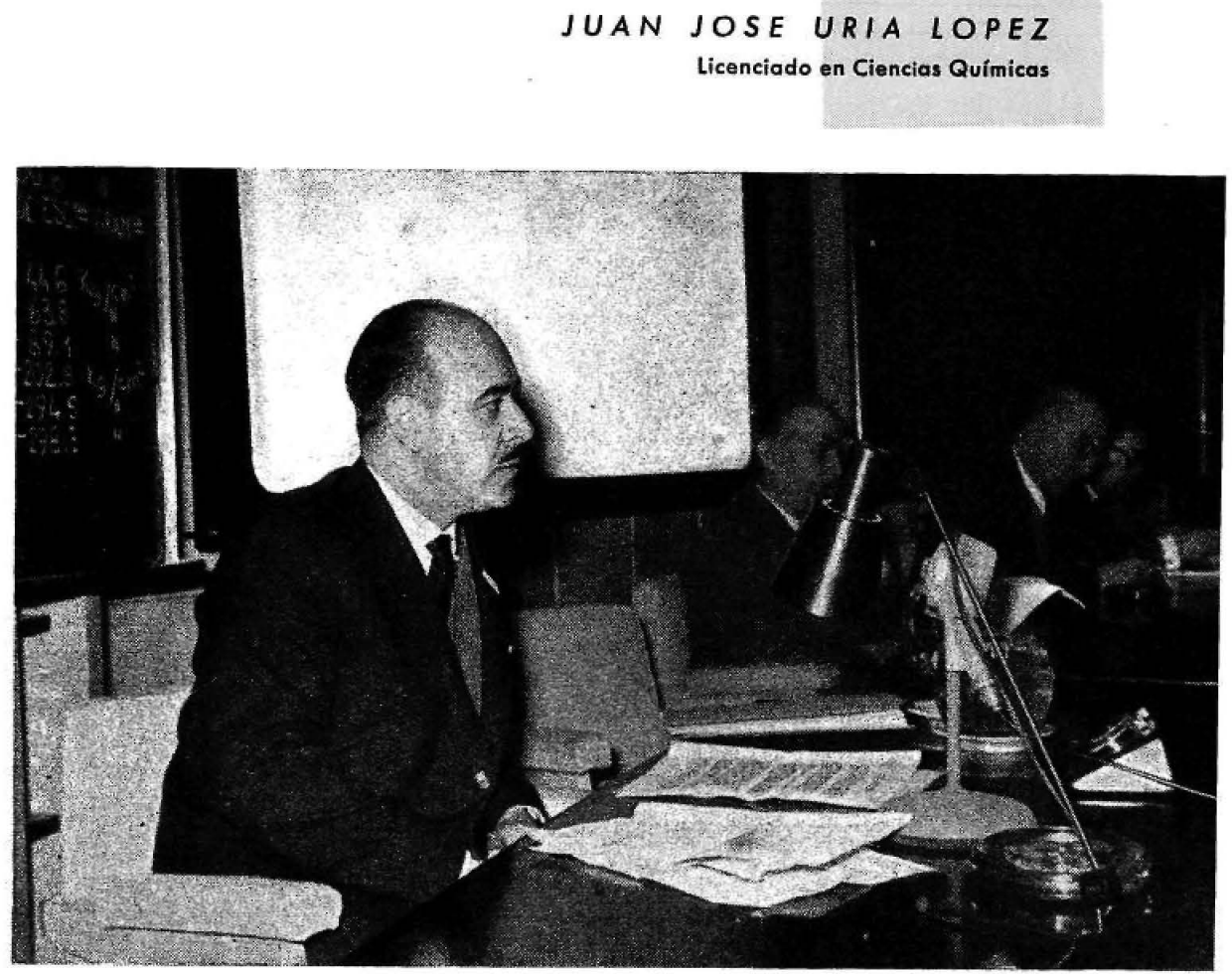

Presentada durante los II Coloquios para Directores y Técnicos de Fábricas de Cemento, la ponencia «Fabricación de Cementos Siderúrgicos», se siguió la correspondiente discusión, proponiéndose en ella la creación de una norma que hiciera posible la fabricación de un cemento Portland con adición de $10 \%$ de escoria granulada como máximo.

Expusimos, por nuestra parte, algunos ensayos que habíamos realizado sobre el particular, indicando que continuábamos los trabajos. Se nos pidió que diéramos a conocer los resultados obtenidos, por lo que aprovechando esta oportunidad los presentamos, esperando dar satisfacción a quienes se interesaron y al mismo tiempo exponemos nuestra pequeña :

«Contribución para el incremento y economía de la fabricación nacional de cemento.»

La producción nacional de escorias de alto horno, subproducto de la industria siderúrgica, excede del medio millón de toneladas. Su aprovechamiento constituye una economía para las Empresas siderúrgicas y, además, contribuye a mejorar la riqueza pública con sus aplicaciones que, aparte de la preparación de áridos de poco peso específico, pómez artificial, lana de escoria, etc., el interés general puede situarse en su utilización y empleo para la fabricación de conglomerantes hidráulicos.

En la actualidad, las fábricas que utilizan las escorias granuladas de alto horno para la fabricación de cementos se hallan situadas en el Levante y Norte de España, empleando, aproximadamente, una cantidad de escorias granuladas que no sobrepasa el $30 \%$ de la producción total, para lo cual se ha instalado en las fábricas los dispositivos necesarios para la fabricación de cementos siderúrgicos. Las escorias sobrantes son arrojadas al mar en embarcaciones acondicionadas al efecto o llegan a constituir enormes escombreras, originando siempre el correspondiente gasto a las fábricas. 
La importancia adquirida por las escorias granuladas en la fabricación de cementos tiene varios aspectos que conviene considerar. Una cuestión importante es la referente a sus propiedades hidráulicas, porque: dilas escorias granuladas de alto homo son o no aptas para fabricar conglomerantes hidráulicos?

Hace setenta años, la adición de escorias al cemento Portland era considerada una adulteración, porque en aquella época algunos fabricantes emplea ban escorias desintegradas en polvo, que es un subproducto desprovisto de valor hidráulico, pero las escorias granuladas de alto horno poseen ya en sí mismas excelentes cualidades conglomerantes (I).

Las escorias granuladas no son puzolanas, sino verdaderos conglomerantes porque intervienen en el fraguado formando silicatos hidratados (2).

La composición química de las escorias para cemento, manifiesta un contenido en cal suficiente para permitir la formación, sin adición alguna, de silicato y aluminato de calcio hídratados; estas escorias son, por consiguiente, verdaderos cementos (Cleretde Langavant), pero también hemos vistos ensayos realizados con escoria fuera de los límites considerados como normales por su composición química que fraguan y se endurecen por estar granuladas (3).

Las escorias de horno alto, vitrificadas mediante un buen enfriamiento, no contienen compuestos mineralógicamente definidos, porque el brusco enfriamiento, al elevar súbitamente la viscosidad, inmoviliza los iones (4). Las escorias granuladas están interiormente, «desordenadas», y la «ordenación» deseada se efectúa por vez primera al fraguado. La energía necesaria se produce mediante la adición de un activador, la molienda fina y el amasado con agua (5).

La importancia de una buena granulación es evidente, si se tiene en cuenta que las escorias cristalinas tienen muy pocas cualidades conglomerantes y las disgregadas por enfriamiento lento ninguna $(6)$.

La energía química de las escorias granuladas es muy grande; la eficacia de la granulación se ha confirmado por las medidas de Prost, que ha encontrado, para el calor de disolución, una diferencia de 119.000 cal. entre los dos estados, en provecho de la escoria granulada (7).

Después de muchos años de controversias ha logrado la escoria de horno alto granulada sustituir todo o en parte al clínker, por actuar en el endurecimiento, porque la escoria granulada tiene un elevado potencial hidráulico, es decir, un enérgico poder conglomerante latente que el clínker $(y$ otras sustancias) ponen de manifiesto al variar las condiciones del medio.

El aprovechamiento de las escorias granuladas como conglomerante hidráulico en la fabricación de cementos, está garantizado por los resultados satisfactorios obtenidos en la práctica de su empleo durante más de cincuenta años (8), habiendo merecido la atención de publicar en disposiciones oficiales las naciones más industrializadas del mundo especificaciones para su empleo, aun las que más han retardado sus aplicaciones hidráulicas $(9$ y 10$)$.

Como resumen, podemos indicar que las escorias de alto horno, finamente molidas, pueden proporcionar cementos de alta calidad al ser mezcladas con cemento Portland (11).

Otra cuestión a considerar es que se tropieza todavía con un temor legendario al empleo de cementos a base de escoria granulada en el hormigón armado, por la supuesta peligrosidad del azufre de sulfuros que contiene (12), y que nunca excede del $2 \%$, basado en opiniones anticuadas lanzadas sobre razonamientos sin experimentación alguna (13). A este respecto, podemos afirmar que el tan sefialado efeclo perjudicial del azufre no ha sido observado en cincuenta años de empleo de la escoria de alto horno, y que todas las exposiciones técnicas lanzadas a este respecto son erróneas (14) y que la opinión carece en absoluto de fundamento (15).

La experiencia establece de una manera indudable que no puede haber transformación de sulfhidrato cálcico con formación de ácidos libres en un medio alcalino y. por tanto, ninguna activación del hierro por destrucción de la película protectora (16). 
En el hierro que ha permanecido durante seis meses y un año en probetas de hormigón fabricado con cementos que contienen escorias granuladas españolas, no se apreció corrosión en ninguna de las probetas $(17)$.

En las experiencias que por nuestra parte hemos efectuado, ha podido observarse que no hay corrosión alguna en plazos hasta de doce años que venimos ensayando esła debatida cuestión.

En resumen, que contrariamente a un prejuicio que tiende a desaparecer, pero todavía tenaz, los cementos a base de escoria granulada aseguran una perfecta protección a las amaduras; por lo cual, los técnicos, convencidos de la inocuidad de los cementos respecto al hierro de las armaduras, la utilizan en hormigón armado como si fuera cemento Portland artificial (18).

En el aspecto técnico-económico, el empleo de la escoria granulada en la fabricación de cemento supone un ahorro considerable de calor y energía, por ser un producto conglomerante que no precisa tratamiento térmico para su empleo inmediato. La utilización de las escorias granuladas supondría un incremento de la producción nacional de cemento y el ahorro de miles de toneladas de carbón, así como energía eléctrica. Puede decirse que cada tonelada de escoria granulada utilizada sustituyendo clínker, economiza alrededor de $200 \mathrm{~kg}$ de carbón (19).

Es de esperar en el progreso nacional que el consumo de cemento aumente, por lo que en el Plan Económico nos encontraremos con dos hechos antagónicos: por una parte, se forzará a economizar carbón y energía eléctrica, y por otra, será necesario aumentar la producción.

El aumento de producción en forma económica va en detrimento de la calidad del cemento, porque el ahorro de carbón conduciría a una clinkerización defectuosa, y el moler el clínker groseramente hace bajar las resistencias, por lo que, conservando la calidad, se puede sustituir parte del clínker por escoria granulada, utilizando al máximo la capacidad de molienda disponible, ahorrándose siempre el molido preliminar y la energía de clínkerización correspondiente.

La escoria granulada por agua retiene de 15 a $30 \%$ de humedad, por lo que exige instalaciones apropiadas y consumo de combustible en cantidad considerable para su secado. Este factor de secado puede evitarse granulando la escoria con aire a presión o por el procedimiento mixto de aire y agua que permite obtener un producto prácticamente seco.

Esta circunstancia económica del ahorro de combustible sería suficiente para aconsejar la utilización de la escoria granulada por cualquiera de los procedimientos en que se obtiene seca, mas también tenemos que considerar la economía de un $25 \%$ del peso inútil del agua en el transporte, muy de tenerse en cuenta en los largos desplazamientos de estos materiales.

$\mathrm{Si}$, además de las ventajas econónicas, consideramos que la calidad de la escoria granulada por aire es prácticamente igual a la granulada por agua, como hemos comprobado después de vatios años de fabricación, habiendo realizado previamente los trabajos correspondientes para el estudio de su estructura y actividad en los laboratorios del Instituto Técnico de la Construcción y del Cemento, así como en otros del extranjero, según las técnicas de Parker y Nurse, el potencial hidráulico según Blondiau y el recuento mictoscópico de los granos vítreos, por lo que estamos satisfechos de los resultados obtenidos, evitándose la posibilidad de una cristalización parcial que un secado a eleveda temperatura de la escoria granulada por agua puede acarrear.

Aunque las instalaciones de los granuladores por aire a presión y agua son costosas y precisan una fuerte amortización por el gran desgaste a que se someten, nosotros estimamos que una escoria perfectamente granulada y seca en condiciones de ser sometida a la molienda para su utilización como conglomerante sustituyendo a una parte de clínker, de cemento Portland, tiene un valor que no excede de la tercera parte del precio de este último, lo que supone una economía que en parte se compensa con la mayor finura de molido que se requiere.

Si a la mitad de la producción de cemento Portland que se fabrica en España se le agregara un $10 \%$ de escorias granuladas secas, podría obtenerse el incremento de un cuarto de tnillón de toneladas de cemento de menor costo y que no precisarían ni un gramo de combustible en su fabricación. 
RESULTADOS OBTENIDOS CON LOS CEMENTOS PREPAR

También se pueden observar los ensayos de los cementos Portlan

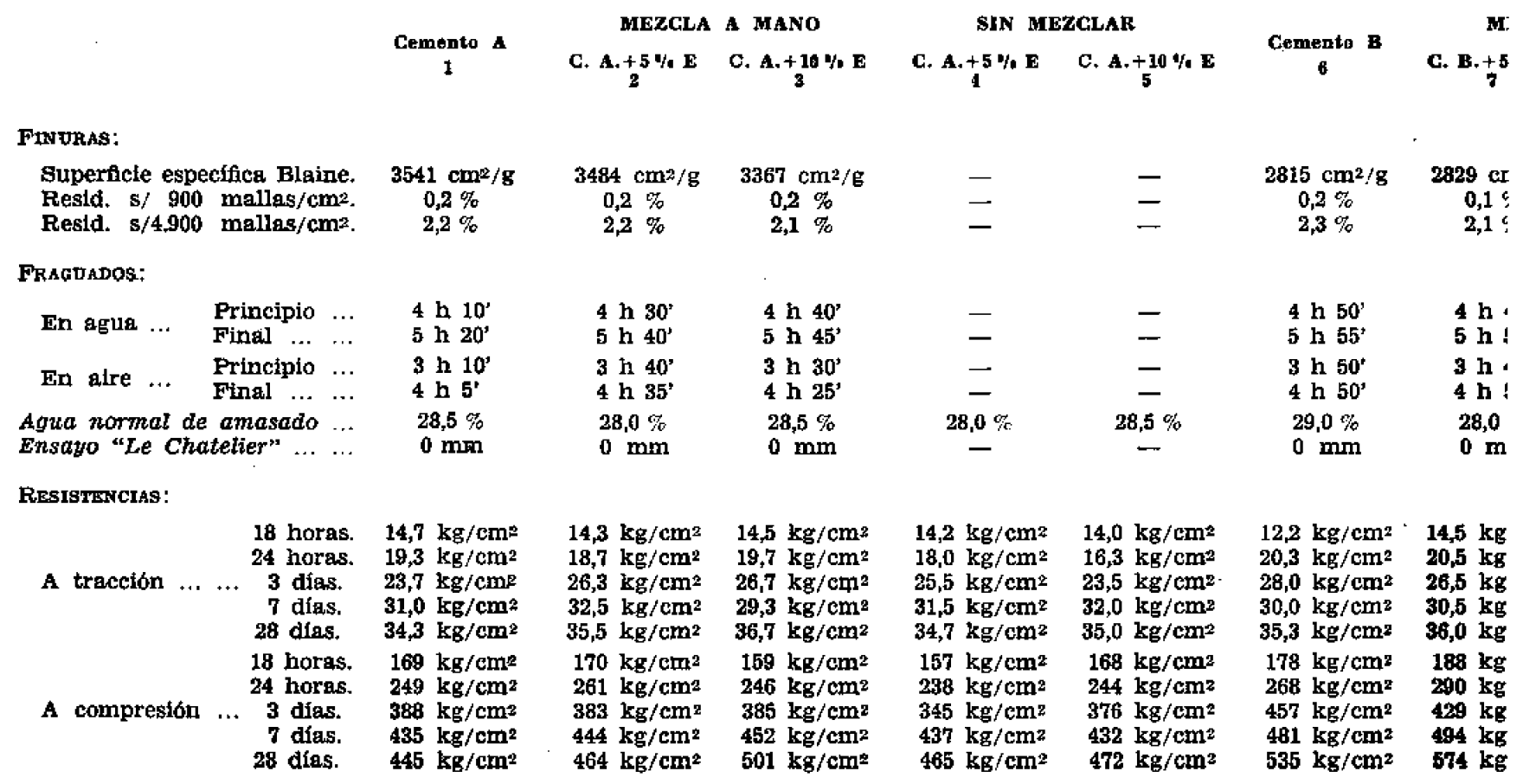

El agregar un $10 \%$ de escoria granulada al cemento Portland está normalizado en Francia (20), sancionado oficialmente allí donde se practica porque hasta el $10 \%$ de contenido en escorias granu. ladas no altera para nada las condiciones del cemento Portland (21).

Claro que esto supone el modificar la estructura orgánica de una fábrica de cemento Portland artificial, porque requiere instalaciones especiales que van desde el movimiento de las materias primas, su secado y preparación, hasta la molienda conjunta de los tres componentes (clínker, escoria granulada y yeso) con sus silos y aparatos dosificadores correspondientes.

Con objeto de simplificar el procedimiento, nosotros ofrecemos una nueva técnica que ya hemos comprobado satisfactoriamente para fabricar cemento Portland con un contenido de escorias granuladas hasta el $10 \%$. Nuestra experiencia y trabajos sobre el particular (22), que coinciden con otros realizados en el extranjero (23), nos han llevado a la conclusión de que una molienda por separado permite regular la superficie específica de los cementos y que para obtener una mezcla conveniente no es necesario la molienda conjunta de los materiales, porque no se precisa un alto grado de mezclado para obtener los mejores resultados. El procedimiento que ofrecemos se basa fundamentalmente en este hecho cierto y comprobado, lo que permitirá agregar escoria granulada en cualquier fábrica de cemento Portland artificial sin cambios fundamentales en su estructuración orgánica de funcionamiento, aprovechando el grado suficiente de mezclado con los productos previamente molidos.

Para ello se ha de moler la escoria granulada seca hasta obtener una superficie específica de 3.400 centímetros cuadrados/gramo Blaine como mínimo (menor de $2 \%$ de residuo sobre 4.900 mallas $/ \mathrm{cm}^{2}$ ), recogerlo en un silo al efecto, no siendo necesario un complicado sistema de básculas automáticas ni de mezcladores para que el producto terminado tenga la calidad deseada, sino que bastará una dosifcación simple por medio de un sencillo procedimiento mecánico conectado a la salida del molino de cemento Portland, siendo suficiente una homogeneización nomal en los silos de cemento para obtener un grado de mezclado satisfactorio. 
[ENIENDO UN $10 \%$ DE ESCORIA GRANULADA MOLIDA

in de escorias, para que puedan ser comparados los resultados

I0<smiles>[13CH3][13CH3]</smiles>

$3 \mathrm{~cm}^{2} / \mathrm{B}$

$0,1 \%$

$2,0 \%$

\section{h $40^{\prime}$ \\ h 50 \\ h 45' \\ h 45' \\ $18,6 \%$}

$3 \mathrm{~mm}$

$\mathrm{kg} / \mathrm{cm}^{2}$ $\mathrm{kg} / \mathrm{cm} 2$

$\mathrm{kg} / \mathrm{cm}^{2}$

$\mathrm{kg} / \mathrm{cm}^{2}$

$\mathrm{kg} / \mathrm{cm}^{2}$

$\mathrm{kg} / \mathrm{cm} 2$

$\mathrm{kg} / \mathrm{cm}^{2}$

$\mathrm{kg} / \mathrm{cm}^{2}$

$\mathrm{k}_{\mathrm{B}} / \mathrm{cm}^{\mathrm{s}}$

i $\mathrm{kg} / \mathrm{cm}=$

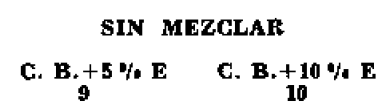

Cemento C
11
$2490 \mathrm{~cm}^{2} / \mathrm{B}$
$0,3 \%$
$5,4 \%$

$3 \mathrm{~h} 40^{\prime}$
$4 \mathrm{~h} 40^{\prime}$
$2 \mathrm{~h} 35$
$3 \mathrm{~h} 25$
$26,5 \%$
$25 \mathrm{~mm}$

$15,0 \mathrm{~kg} / \mathrm{cm}^{2}$

$22,5 \mathrm{~kg} / \mathrm{cm}^{2}$

$29,2 \mathrm{~kg} / \mathrm{cm}^{2}$

$33,7 \mathrm{~kg} / \mathrm{cm}^{2}$

$36,0 \mathrm{~kg} / \mathrm{cm}^{2}$

$244 \mathrm{~kg} / \mathrm{cm}^{2}$

$292 \mathrm{~kg} / \mathrm{cm}^{2}$

$420 \mathrm{~kg} / \mathrm{cm}^{2}$

$508 \mathrm{~kg} / \mathrm{cm}$ ?

$565 \mathrm{~kg} / \mathrm{cm}^{2}$
10

-

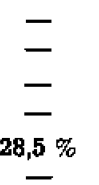

$14,2 \mathrm{~kg} / \mathrm{cm}^{2}$

$21,5 \mathrm{~kg} / \mathrm{cm}^{2}$

$28,7 \mathrm{~kg} / \mathrm{cm}^{2}$

$32,8 \mathrm{~kg} / \mathrm{cm}^{2}$

$35,5 \mathrm{~kg} / \mathrm{cm}^{2}$

$242 \mathrm{~kg} / \mathrm{cm}^{2}$

$276 \mathrm{~kg} / \mathrm{cm}^{2}$

$435 \mathrm{~kg} / \mathrm{cm}^{2}$

$499 \mathrm{~kg} / \mathrm{cm}^{2}$

$53 \mathrm{~B} \mathrm{~kg} / \mathrm{cm}^{2}$

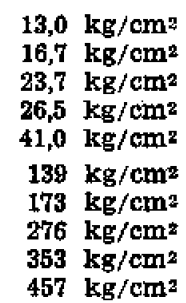

$13,0 \mathrm{~kg} / \mathrm{cm}^{2}$

$26,5 \mathrm{~kg} / \mathrm{cm}^{2}$

$41,0 \mathrm{~kg} / \mathrm{cm} 2$

$139 \mathrm{Kg} / \mathrm{cm}^{2}$

$276 \mathrm{~kg} / \mathrm{cm}^{2}$

$45 \mathrm{~kg} / \mathrm{cm}^{2}$
kB/cm
MEZCLA a MANO

$\begin{array}{ll}\text { C. } C .+5 \%+\mathrm{B} & \text { C. C. }+14 \% \mathrm{E}\end{array}$

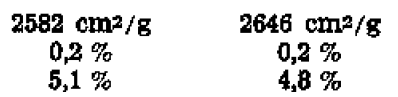

2 h 50'

$3 \mathrm{~h} 40^{\circ}$

$2 \mathrm{~h} \mathrm{25}$

3 h $10^{*}$

$28,0 \%$

$7 \mathrm{~mm}$

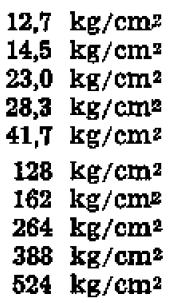

$12,7 \mathrm{~kg} / \mathrm{cm}^{2}$

14,5 $\mathrm{kg} / \mathrm{cm}^{2}$

$28,3 \mathrm{~kg} / \mathrm{cm}^{2}$

$41,7 \mathrm{~kg} / \mathrm{cm}^{2}$

$12 \mathrm{~B} \mathrm{Ke} / \mathrm{cm}^{2}$

$162 \mathrm{~kg} / \mathrm{cm}^{2}$

$388 \mathrm{~kg} / \mathrm{cm}^{2}$

$524 \mathrm{~kg} / \mathrm{cm}^{2}$
3 h 15'

$4 \mathrm{~h} 20^{\prime}$

$2 \mathrm{~h} 20^{\prime}$

$3 \mathrm{~h} 15^{\prime}$

$28,5 \%$

$0 \mathrm{~mm}$

$12,7 \mathrm{~kg} / \mathrm{cm}^{2}$

$15,3 \mathrm{~kg} / \mathrm{cm}^{2}$

$22,8 \mathrm{~kg} / \mathrm{cm}^{2}$

$27,0 \mathrm{Kg} / \mathrm{cm}^{2}$

$42,0 \mathrm{~kg} / \mathrm{cmo}^{2}$

$131 \mathrm{~kg} / \mathrm{cm}^{2}$

$169 \mathrm{~kg} / \mathrm{cm}^{2}$

$298 \mathrm{~kg} / \mathrm{cm}^{2}$

$381 \mathrm{~kg} / \mathrm{cm}^{2}$

$513 \mathrm{~kg} / \mathrm{cm}^{2}$
SIN MEZGLAR

C. C. $+5 \% E \quad$ C. C. $+10 \%$ B

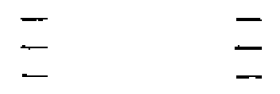

-
-
$28,0 \%$

-
-
$28,5 \%$ $\begin{array}{rl}11,0 & \mathrm{~kg} / \mathrm{cm}^{2} \\ 14,7 & \mathrm{Kg} / \mathrm{cm}^{2} \\ 22,5 & \mathrm{~kg} / \mathrm{cm}^{2} \\ 30,3 & \mathrm{~kg} / \mathrm{cm}^{2} \\ 39,0 & \mathrm{~kg} / \mathrm{cm}^{2} \\ 112 & \mathrm{~kg} / \mathrm{cm}^{2} \\ 153 & \mathrm{~kg} / \mathrm{cm}^{2} \\ 269 & \mathrm{~kg} / \mathrm{cm}^{2} \\ 406 & \mathrm{~kg} / \mathrm{cm}^{2} \\ 547 & \mathrm{~kg} / \mathrm{cm}^{2}\end{array}$

$12,5 \mathrm{~KB} / \mathrm{cm}^{2}$

$16,3 \mathrm{kB} / \mathrm{cm}^{2}$

$28,3 \mathrm{~kg} / \mathrm{cm}^{2}$

$27,5 \mathrm{~kg} / \mathrm{cm}^{2}$

$42,0 \mathrm{Kg} / \mathrm{cm}^{2}$

$127 \mathrm{~kg} / \mathrm{cm}^{2}$

$168 \mathrm{~kg} / \mathrm{cm}^{2}$

$273 \mathrm{~kg} / \mathrm{cm}^{2}$

$371 \mathrm{~kg} / \mathrm{cm} 7$

618 $\mathrm{kg} / \mathrm{cm}^{2}$

Nosotros hemos comprobado el procedimiento que acabamos de exponer realizando ensayos con varios cementos Portland de distintas fábricas adquiridos en el mercado.

Los ensayos realizados han consistido en mezclar cemento Portland con el 5 y $10 \%$ de escoria granulada molida, unos mezclando los componentes a mano; para lo cual se colocan unos sobre otros en forma de pila, se divide y se deshace, se vuelve a rehacer la pila, y así sucesivamente durante tres minutos. Esta mezcla manual resulta incompatible para una fabricación, pero las pruebas se han realizado con objeto de comprobar la calidad de una mezcla hecha en las condiciones más inferiores. Otra parte no se mezcló previamente, sino que fueron colocados en la artesa el cemento Portland, la escoria molida y la arena normal de ensayos, a todo lo cual se le agregó el agua de una sola vez, realizándose el amasado normal del ensayo. Los resultados obtenidos se exponen en el cuadro que se adjunta.

Los números 1, 6 y 11 corresponden al ensayo del cemento Portland normal correspondiente.

Los números 2,7 y 12 corresponden a los cementos Portland que contienen $5 \%$ de escoria granulada molida, mezclados a mano.

Los números 3,8 y 13 corresponden a los cementos Portland que contienen $10 \%$ de escoria granulada molida, mezclados a mano.

Los números 4, 9 y 14 corresponden a los cementos Portland que contienen $5 \%$ de escoria granulada molida, sin mezcla previa.

Los números 5, 10 y 15 corresponden a los cementos Portland que contienen $10 \%$ de escoria granulada molida, sin mezcla previa.

Como final exponemos los resultados obtenidos sobre dos cementos Portland artificiales de buena calidad adquiridos en el mercado, realizándose los ensayos de acuerdo con las nuevas normas vigentes, es decir, con mortero comptesto de $1.500 \mathrm{~g}$ de arena normalizada, $500 \mathrm{~g}$ de cemento y $250 \mathrm{c}$. c. de agua. 
Ensayo del cemento Portland $« \mathbf{X} »$.

Resistencias:
A flexotracción, a 3 días: $49,5 \mathrm{~kg} / \mathrm{cm}^{2}$
7 días: $66,7 \mathrm{~kg} / \mathrm{cm}^{2}$
A compresión, a 3 días: $217 \mathrm{~kg} / \mathrm{cm}^{2}$
7 días: $306,5 \mathrm{~kg} / \mathrm{cm}^{2}$
28 días: $91,8 \mathrm{~kg} / \mathrm{cm}^{2}$
28 días: $403 \mathrm{~kg} / \mathrm{cm}^{2}$

Ensayo del cemento Portland $\alpha X$ que contiene $10 \%$ de escoria granulada. Resistenclas :
A flexotracción, a 3 días: $49,4 \mathrm{~kg} / \mathrm{cm}^{2}$
7 días: $66,1 \mathrm{~kg} / \mathrm{cm}^{2}$
$\begin{array}{ll}\text { A compresión, a } & 3 \text { días: } 217 \mathrm{~kg} / \mathrm{cm}^{2} \\ & 7 \text { dias : } 299,1 \mathrm{~kg} / \mathrm{cm}^{2}\end{array}$
28 días: $91,6 \mathrm{~kg} / \mathrm{cm}^{2}$
28 días: $406,3 \mathrm{~kg} / \mathrm{cm}^{2}$

Ensayo del cemento Portland $\alpha \mathbf{Y}$.

Resistenclas:
A flexotracción, a 3 días: $45,1 \mathrm{~kg} / \mathrm{cm}^{2}$
7 días: $59,4 \mathrm{~kg} / \mathrm{cm}^{2}$
28 días: $85,8 \mathrm{~kg} / \mathrm{cm}^{2}$
A compresión, a 3 días: $191,5 \mathrm{~kg} / \mathrm{cm}^{2}$
7 días: $286,7 \mathrm{~kg} / \mathrm{cm}^{2}$
28 días: $390,6 \mathrm{~kg} / \mathrm{cm}^{2}$

Ensayo del cemento Portland "Y⿱ $)$ que contiene $10 \%$ de escoria granulada.

Resistenclas :
A flexotracción, a 3 días: $44,6 \mathrm{~kg} / \mathrm{cm}^{2}$
7 días: $63,8 \mathrm{~kg} / \mathrm{cm}^{2}$
28 días: $89,1 \mathrm{~kg} / \mathrm{cm}^{2}$
A compresión, a 3 días: $202,8 \mathrm{~kg} / \mathrm{cm}^{2}$
7 días: $294,5 \mathrm{~kg} / \mathrm{cm}^{2}$
28 días : $398,3 \mathrm{~kg} / \mathrm{cm}^{2}$

El valor de los resultados obtenidos puede ser comprobado por quienes se interesen en el procedimiento; nuestra exposición lo mismo que en otros trabajos que anteriormente hemos presentado y que coinciden con los de otros especialistas del extranjero, es demasiado clara para que pueda ser interpretado como un interés comercial o industrial, porque la guía de nuestros trabajos es el engrandecimiento y prosperidad de nuestra Patria con estas pequeñas aportaciones.

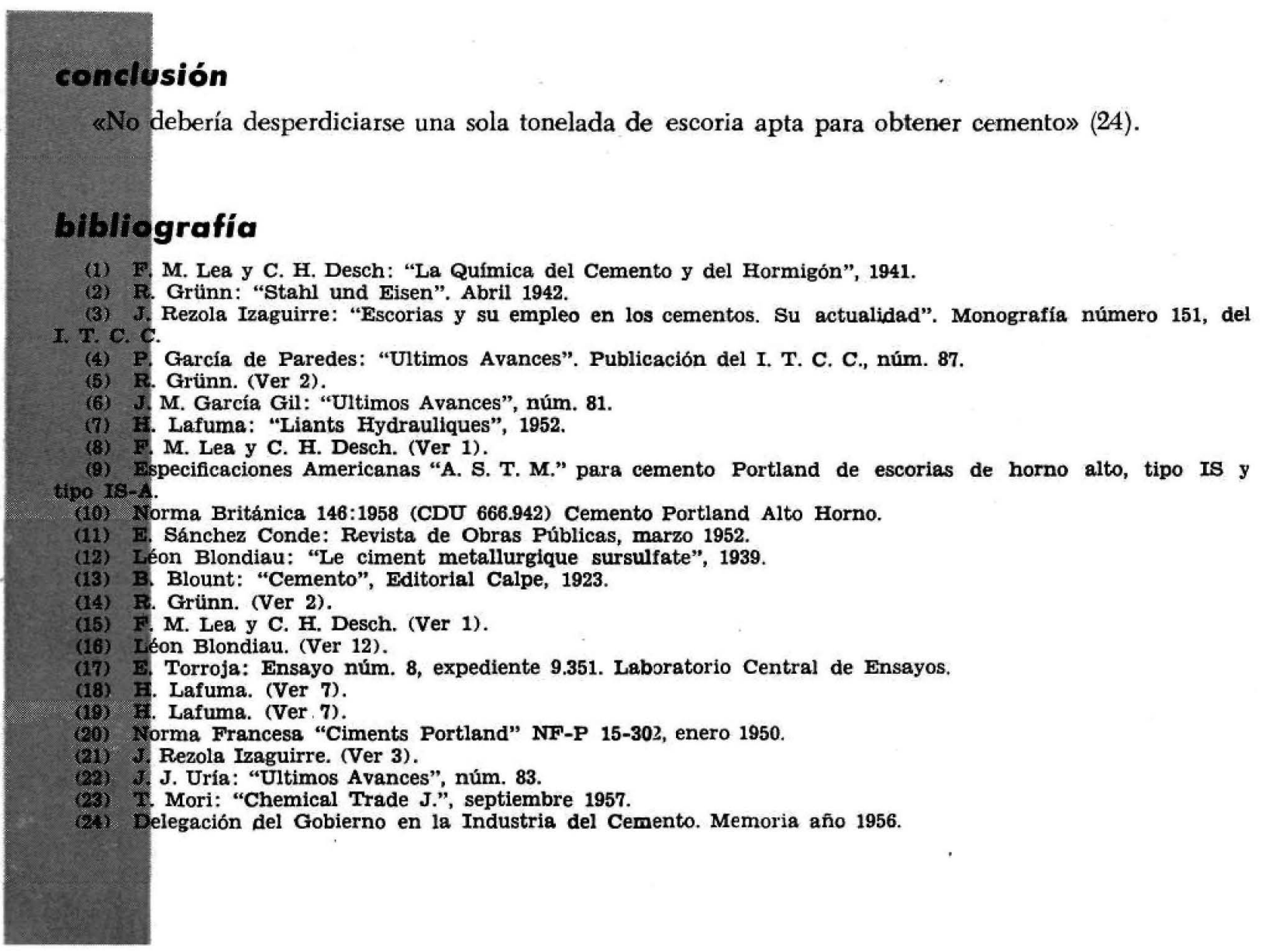

